\title{
Leprosy with Necrosis in Granulomatous Reaction
}

Case 1: A 43 year-old, white man presented with a four month-history of six plaques well-circumscribed. Laboratory results, including several serological tests, as well as a radiological investigation, were either negative or normal. The Mitsuda reaction was positive (9 $\mathrm{mm}$ ). He was put on prednisone therapy and multi-drug therapy (MDT) with subsequent healing of the cutaneous lesions and improvement of the neuritis in about six months. The diagnostic hypothesis was reactional tuberculoid leprosy (Figure 1).

Case 2: A 23 year-old, mulatto man with a two-year history of prednisone treatment for bouts of nodules appearing over a macula who had been on treatment for tuberculoid leprosy with MDT-PB. Laboratory data, which included a search for other infectious diseases (HIV, HTLV-1 and 2, hepatitis B and C, syphilis, tuberculosis, and chest X-ray) was normal/negative. However his tuberculosis skin test (PPD) was strongly positive $(30 \mathrm{~mm})$, and the Mitsuda reaction was also positive $(7 \mathrm{~mm})$. A biopsy revealed a chronic granulomatous dermatitis with caseous necrosis. Although a search for $M$. tuberculosis was negative by immunehistochemistry and PCR, he received conventional anti-TB therapy, with improvement of the nodules and the infiltrative lesions. Hypotheses: Tuberculoid leprosy in one reaction with caseous necrosis or Tuberculosis and Tuberculoid leprosy?

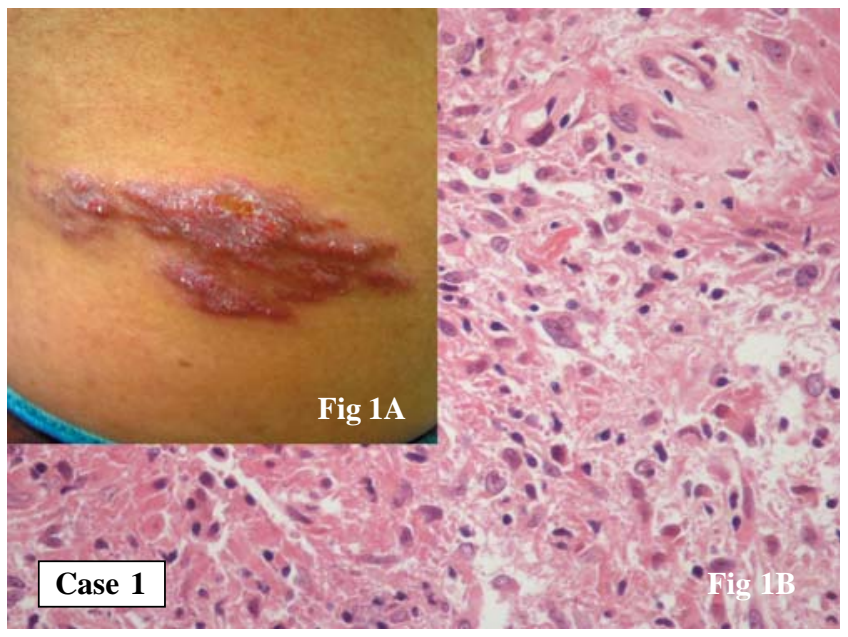

Figure 1 A. A well-circumscribed ulcerated and infiltrated patch, with altered temperature sensitivity; Figure 1B. HE staining shows a chronic granulomatous (CG) dermatitis with fibrinoid necrosis and a thickened nerve. Faraco and Grocott negative.

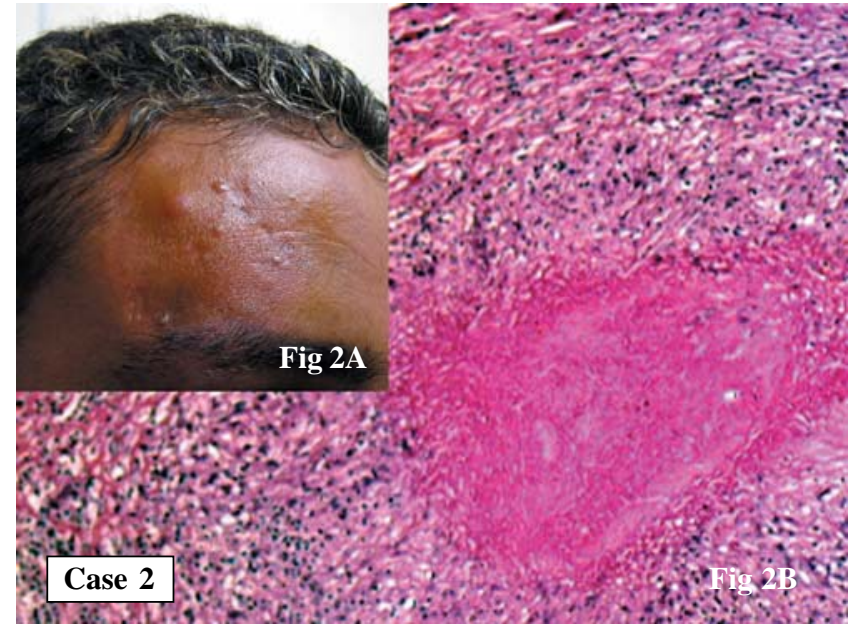

Figure 2A. A fronto-temporal erytemathous and infiltrative $4 \mathrm{~cm}$ patch, with well-defined borders and nodulo-cystic lesions larger than 0.5 to $2 \mathrm{~cm}$, with purulent secretion; Figure 2B. HE: Chronic granulomatosus reaction with extensive caseous necrosis and neuritis on dermis and hypodermis.

Discussion: Various infectious/inflammatory diseases are associated with necrosis [1-3]. Caseous lesions are frequently seen in tuberculosis but are rare in leprosy granulomas [4]. In case 1, the corticotherapy may have accelerated the healing of the ulcers and limited the fibrinoid necrosis, avoiding the deleterious effects of exacerbated host cell-mediated immunity [5]. In case 2, the two-year persisting caseous necrosis could have been due to a reinfection partially controlled by rifampicine. The stronglypositive PPD might be due to a cross reactivity between $M$. tuberculosis and M. leprae. However, neural involvement, the histopathology, and the lack of other pathogens, point to a leprosy granulomatous reaction [6-8]. These cases illustrate the difficulties in establishing a definitive diagnosis of a chronic infectious disease, when it is accompanied by a strong cellular immunity response, usually resulting in complete elimination of the invading organisms [9-10]. This difficulty emphasizes the need for careful anamnesis and dermato-neurological examination, looking for areas of anesthesia and neural thickening, to establish the diagnosis of leprosy, while we await improvement in the laboratory diagnostic techniques for mycobacteria.

Maria Ângela Bianconcini Trindade ${ }^{1,2}$, Brandt H. ${ }^{1}$, Teixeira R. ${ }^{1}$, Sotto M.N. ${ }^{1}$ and Fleury R.N. ${ }^{3}$

${ }^{1}$ Dermatology, Clinical Hospital, Medicine Faculty of São Paulo University; ${ }^{2}$ Health Institut and 3Lauro Souza Lima Institut, Department of Health State of São Paulo; São Paulo, SP, Brazil

E-mail: angelatrindade@uol.com.br

\section{References}

1. James D.G. A clinicopathological classification of granulomatous disorders. Postgrad Med J 2000;76;457-65.

2. Rabinowitz L.O., Zaim M.T. A clinicopathologic approach to granulomatous dermatoses. J Am Acad Dermatol 1996;35:588-600.

3. The 69th annual meeting symposium. II: Mechanism of necrotizing granuloma formation and its function. Kekkaku 1994;69(10):627-49.

4. Ridley D.S., Radia K.B. The histological course of reactions in borderline leprosy and their outcome. Int $\mathrm{J}$ Lepr Other Mycobact Dis 1981;49(4):383-92.
5. Job C.K. Pathology and pathogenesis of leprous neuritis; a preventable and treatable complication. Int J Lepr Other Mycobact Dis 2001;69(2 Suppl):S19-29.

6. Job C.K. Nerve in reversal reaction. Indian J Lepr 1996;68(1):43-7.

7. Antia N.H., et al. Plasma cells in caseous necrosis of nerves in leprosy. Lepr Rev 1985;56(4):331-5.

8. Chandi S.M., et al. Segmental necrotizing granulomatous neuritis of leprosy. Int J Lepr Other Mycobact Dis 1980;48(1):41-7.

9. Moschella SL. The lepra reaction with necrotizing skin lesions. A report of six cases. Arch Dermatol 1967;95(6):565-75.

10. Nirmala V., et al. Tuberculoid leprosy and tuberculosis skin: a comparative histopathological study. Lepr India 1977;49(1):65-9.

The Brazilian Journal of Infectious Diseases (www.bjid.com.br) 2008;12(5):460. ㄷ 2008 by The Brazilian Journal of Infectious Diseases and Contexto Publishing. All rights reserved. 